International Journal of Modern Physics D, (C) World Scientific Publishing Company

\title{
INITIAL OPERATION OF THE INTERNATIONAL GRAVITATIONAL EVENT COLLABORATION
}

\author{
G.A. PRODI ${ }^{a *}$ I.S. HENG,${ }^{b * \dagger}$ Z.A. ALLEN,${ }^{b}$ P. ASTONE,${ }^{c}$ L. BAGGIO,${ }^{d}$ M. BASSAN,$e^{e, f}$ \\ D.G. BLAIR,${ }^{g}$ M. BONALDI ${ }^{h}$ P. BONIFAZI,${ }^{i, c}$ P. CARELLI,${ }^{j}$ M. CERDONIO,$^{d}$ \\ E. COCCIA,${ }^{e, f} \mathrm{~L}$. CONTI ${ }^{d}$ C. COSMELLI,${ }^{k, c} \mathrm{~V}$. CRIVELLI VISCONTI ${ }^{d}$ S. D'ANTONIO,${ }^{l}$ \\ V. FAFONE ${ }^{l}$ P. FALFERI,${ }^{h}$ P. FORTINI, ${ }^{m}$ S. FRASCA,${ }^{k, c}$ W.O. HAMILTON,${ }^{b}$ \\ E.N. IVANOV,${ }^{g}$ W.W. JOHNSON,${ }^{b}$ C.R. LOCKE,${ }^{g}$ A. MARINI,${ }^{l}$ V. MARTINUCCI,${ }^{a}$ \\ E. MAUCELI,${ }^{l}$ M.P. McHUGH,${ }^{b}$ R. MEZZENA,${ }^{a}$ Y. MINENKOV,${ }^{f}$ I. MODENA,${ }^{e, f}$ \\ G. MODESTINO,$^{l}$ A. MOLETI ${ }^{e, f}$ A. ORTOLAN,${ }^{n}$ G.V. PALLOTTINO,${ }^{k, c}$ G. PIZZELla $,{ }^{e}, l$ \\ E. ROCCO ${ }^{d}$ F. RONGA,$^{l}$ F. SALEMI ${ }^{k}$ G. SANTOSTASI, ${ }^{b}$ L. TAFFARELLO ${ }^{o}$ \\ R. TERENZI,${ }^{i, f}$ M.E. TOBAR, ${ }^{g}$ G. VEDOVATO,$^{n}$ A. VINANTE ${ }^{a}$ M. VISCO,${ }^{i, f}$ S. VITALE ${ }^{a}$ \\ L. VOTANO ${ }^{l}$ J.P. ZENDRI ${ }^{\circ}$ \\ a Dipartimento di Fisica, Università di Trento, and I.N.F.N., Gruppo Collegato di Trento, \\ I-38050 Povo, Trento, Italy \\ ${ }^{b}$ Department of Physics and Astronomy, Louisiana State University, \\ Baton Rouge, Louisiana 70803 \\ c I.N.F.N., Sezione di Roma1, P.le A.Moro 2, \\ I-00185, Roma, Italy \\ d Dipartimento di Fisica, Università di Padova, and I.N.F.N., Sezione di Padova, \\ Via Marzolo 8, 35131 Padova, Italy \\ e Dipartimento di Fisica, Università di "Tor Vergata", Via O.Raimondo, \\ I-00173 Roma, Italy \\ f I.N.F.N. Sezione di Roma2, Via O.Raimondo, \\ I-00173 Roma,Italy \\ $g$ Department of Physics, University of Western Australia, \\ Nedlands, WA 6907 Australia \\ ${ }^{h}$ Centro di Fisica degli Stati Aggregati, I.T.C.- C.N.R., and I.N.F.N., Trento, \\ I-38050 Povo, Trento, Italy \\ ${ }^{i}$ Istituto Fisica Spazio Interplanetario, C.N.R., Via Fosso del Cavaliere, \\ I-00133 Roma, Italy \\ j Dipartimento di Fisica, Università de L'Aquila, and I.N.F.N., \\ L'Aquila, Italy \\ ${ }^{k}$ Dipartimento di Fisica, Università "La Sapienza", P.le A.Moro 2, \\ I-00185, Roma, Italy \\ ${ }^{l}$ Laboratori Nazionali di Frascati, Istituto Nazionale di Fisica Nucleare, Via E.Fermi 40, \\ I-00044, Frascati, Italy \\ $m$ Dipartimento di Fisica, Università di Ferrara, and I.N.F.N., Sezione di Ferrara \\ I-44100 Ferrara, Italy \\ ${ }^{n}$ Laboratori Nazionali di Legnaro, Istituto Nazionale di Fisica Nuclare, \\ 35020 Legnaro, Padova, Italy \\ o Sezione di Padova, Istituto Nazionale di Fisica Nucleare, via Marzolo 8,
}

\footnotetext{
*presenting authors

${ }^{\dagger}$ previously at: Department of Physics, University of Western Australia, Nedlands, WA 6907
} 
I-35131 Padova, Italy

Received (received date)

Revised (revised date)

\begin{abstract}
The International Gravitational Event Collaboration, IGEC, is a coordinated effort by research groups operating gravitational wave detectors working towards the detection of millisecond bursts of gravitational waves. Here we report on the current IGEC resonant bar observatory, its data analysis procedures, the main properties of the first exchanged data set. Even though the available data set is not complete, in the years 1997 and 1998 up to four detectors were operating simultaneously. Preliminary results are mentioned.
\end{abstract}

\title{
1. The International Gravitational Event Collaboration
}

One of the most relevant scientific objectives for resonant detectors of gravitational waves (gw) is the search for short gw bursts, emitted during the gravitational collapse of stars or the final evolution of coalescing binaries. 1 To ensure the confidence of a detection, it is necessary to compare the observations made by multiple detectors with uncorrelated noise. This has already been done in the past years among pairs of cryogenic detectors with common time periods of observation of about a semester.2. 3 A few days of observation have been reported also for three simultaneously operating detectors. In these attempts, the gw search consisted of a time coincidence analysis among the candidate signals reported by the different detectors and no statistically significant excess of coincidences was found.

An increase of the number of cryogenic resonant detectors in simultaneous operation in recent years has greatly improved the chance of making a confident detection of gw bursts. In fact, the International Gravitational Event Collaboration, IGEC, currently consists of the research groups operating the five cryogenic bar detectors ALLEGRO, AURIGA, EXPLORER, established in July 1997 with an agreement for setting up a common search for gravitational wave bursts of duration of the order of $1 \mathrm{~ms}$. This agreement sets the guidelines for the data exchange procedure among the participating groups and the IGEC scientific policy, whose most relevant aspects are:

- each group has responsibility to make available to IGEC its list of candidate gravitational wave events,

- a unanimous agreement of the member groups is required to make public the results based on the IGEC data exchange,

- IGEC is open to new data taking research groups.

In 1999 the first IGEC analysis of the 1997-1998 data was performed and some initial results will be presented in the following sections. At the time of this analysis, not all the 1997-1998 data had been exchanged. Despite the incomplete data set, the simultaneous operation of four gravitational wave detectors was achieved for the first time. 


\subsection{Data exchange protocol}

The IGEC data exchange procedure is aimed at searching for coincident excitations at different detectors. For each detector, a list of candidate events, each describing a $\delta$-like gravitational wave excitation of the detector, is provided by the corresponding research group. The IGEC protocol requires that in each list the candidate event rate be at most of the order of 100/day, to limit the expected rate of accidental coincidences. Currently, the research groups have been exchanging event lists relative to the past three years, but a future goal is to implement an automatic exchange day by day.

The event lists are then made available to the IGEC collaboration as files under a common protocol, 1 open for future extensions. Under this protocol, it is mandatory for each detector to provide the minimum set of information needed to describe a $\delta$-like gw excitation of the detector for each event; namely its Universal Time

of arrival, the Fourier component $H_{O}$ of its amplitude in $\mathrm{Hz}^{-1}$ and the detector noise level at that time. Another mandatory requirement is the declaration of the effective observation time of each detector, so that the IGEC observation time can be calculated. Optional information, such as the time of threshold crossing of the detector output, the duration of the event and its statistical compliance to a $\delta$-like gw excitation, can also be exchanged.

\subsection{IGEC gravitational wave observatory}

The relevant parameters of the five resonant bar detectors of the IGEC observatory in the years 1997-1998 are summarised in Table 1. The detectors are sensitive to gw signals in a typical bandwidth of the order of $1 \mathrm{~Hz}$ around each one of the two resonances of the detector, which are close to $700 \mathrm{~Hz}$ for NIOBE and close to $900 \mathrm{~Hz}$ for all other detectors. The relationship between the Fourier amplitude $H_{0}$, averaged on the two resonant frequencies of the detector, and the energy $E_{s}$ deposited by the g.w. burst on the bar, is given by:

$$
H_{0}=\frac{1}{4 L_{b a r} \nu_{0}^{2}} \sqrt{E_{s} / M_{b a r}}
$$

where $L_{b a r}$ is the bar length, $M_{b a r}$ its mass, $\nu_{0}$ the mean of the detector resonance frequencies.

The typical thresholds used for selecting the events in the 1997-1998 burst search have been in the range $H_{0} \simeq 2-6 \times 10^{-21} \mathrm{~Hz}^{-1}$. The corresponding strain amplitude of the gw can be computed assuming a model for the burst shape: for the conventional $\sim 1 \mathrm{~ms}$ burst, the Fourier component $H_{0}$ should be multiplied by $10^{3} \mathrm{~Hz}$ to get the maximum strain amplitude $h$.

To maximise the chances of a coincidence detection, the bars have been oriented to be approximately parallel to one another. Neglecting the polarisation effects, the gw amplitude at the detector is $H_{0}=H_{g w} \sin ^{2} \theta(t)$, where $H_{g w}$ is the incident gw amplitude and $\theta(t)$ is the angle between the bar axis and the direction of the 
Initial Operation of the International Gravitational Event Collaboration

Table 1. Main characteristics of the five resonant bar detectors in 1997-1998. The reported misalignment is the angle between the bar axis and an optimal direction whose overall misalignment from the detectors is minimal. $Q_{ \pm}$is the typical quality factor of the resonances

\begin{tabular}{lccccc}
\hline ANTENNA & ALLEGRO & AURIGA & EXPLORER & NAUTILUS & NIOBE \\
\hline Material & $A L 5056$ & $A L 5056$ & $A L 5056$ & $A L 5056$ & $N b$ \\
Mass $[\mathrm{kg}]$ & 2296 & 2230 & 2270 & 2260 & 1500 \\
Length $[\mathrm{m}]$ & 3.0 & 2.9 & 3.0 & 3.0 & 2.75 \\
Mode - $[\mathrm{Hz}]$ & 895 & 912 & 905 & 908 & 694 \\
Mode $+[\mathrm{Hz}]$ & 920 & 930 & 921 & 924 & 713 \\
$Q_{ \pm}\left[10^{6}\right]$ & 2 & 3 & 1.5 & 0.5 & 20 \\
Temp. $[\mathrm{K}]$ & 4.2 & 0.2 & 2.6 & 0.1 & 5.0 \\
Longitude & $268^{\circ} 50^{\prime} E$ & $11^{\circ} 56^{\prime} 54^{\prime \prime} E$ & $6^{\circ} 12^{\prime} E$ & $12^{\circ} 40^{\prime} 21^{\prime \prime} E$ & $115^{\circ} 49^{\prime} E$ \\
Latitude & $30^{\circ} 27^{\prime} N$ & $45^{\circ} 21^{\prime} 12^{\prime \prime} N$ & $46^{\circ} 27^{\prime} N$ & $41^{\circ} 49^{\prime} 26^{\prime \prime} N$ & $31^{\circ} 56^{\prime} S$ \\
Azimuth & $40^{\circ} \mathrm{W}$ & $44^{\circ} E$ & $39^{\circ} E$ & $44^{\circ} E$ & $0^{\circ}$ \\
Misalignment $[\mathrm{deg}]$ & 9 & 4 & 2 & 3 & 29 \\
\hline
\end{tabular}

source. In this configuration of the observatory, the relative misalignments reported in Tab. 1 for ALLEGRO, AURIGA, EXPLORER and NAUTILUS disperse their $\sin ^{2} \theta(t)$ responses by at most a few $\%$, while for NIOBE the dispersion is up to a few tenths. Figure 1 shows as an example the resulting amplitude efficiency for the observation of the Galactic Center as the Earth rotates. Since their values of $\sin ^{2} \theta(t)$ are simultaneously above 0.7 for about $60 \%$ of the time, we point out that this configuration of the observatory ensures a rather good and coherent coverage of the central galactic mass during time.

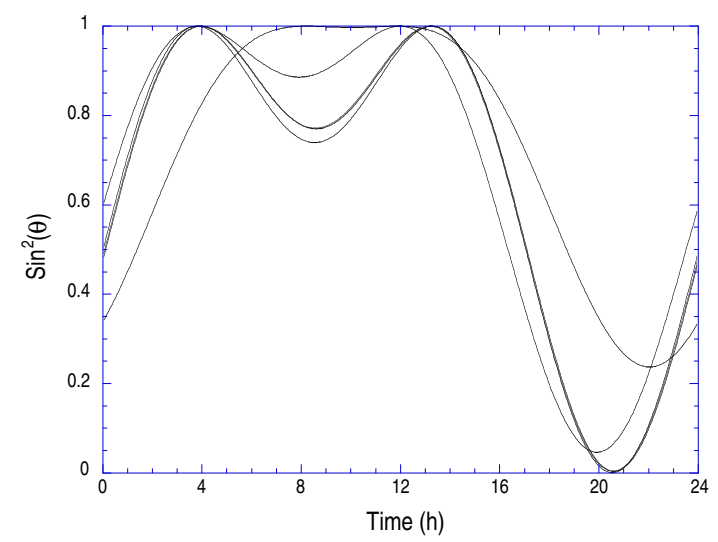

Fig. 1. Amplitude response $\sin ^{2} \theta(t)$ of the five IGEC detectors versus Universal Time for signals incoming from the Galactic Center during one day.

\section{The Data Set}

Each IGEC group independently developed a data acquisition and an optimum 
filtering procedure for a $\delta$-like gw excitation. These procedures differ greatly in the methods in particular some of them filter for the energy released in the bar (ALLEGRO, NIOBEB) while the others filter for the amplitude and phase of the strain excitation of the bar (AURIGA 10 EXPLORER and NAUTILUS11). The output correlation time of the filters ranges from a few tenths up to a few seconds.

A search for maxima on the filter outputs is then used to identify the time and amplitude of the candidate events, which are exchanged only if their amplitude exceeds a selected threshold relative to the noise level. For the current detectors, these thresholds span in the range of signal-to-noise ratio $S N R \simeq 3-5$ in amplitude. Of the events overcoming the threshold, some are rejected as spurious by different methods. AURIGA implements a $\chi^{2}$ test on the compliance of the single detected excitations with the expected template of a gw burst. 22 In fact, the filtering procedure implemented is equivalent to a maximum likelihood fit of a signal model to the data and the goodness of the fit is statistically tested for each candidate event; those events not passing the test are rejected. All the other detectors implement spurious signals rejection using sensors of ambient disturbances. In addition, EXPLORER and NAUTILUS reject the events when the local noise is above a certain threshold.

The effective observation times for each detector has been defined by vetoing, $a$ priori, time periods of detector maintenance or malfunctions and times when the detector was excited by the local environment as determined by the local experimentalists. After filtering, AURIGA implements a second level of vetoes a posteriori to reject further periods of unsatisfactory performance due to a lack of self consistency of its data analysis, that is when its noise fails to be compliant with the modeled one used to build its filtering procedure 13

Table 2. Left: total observation time $T_{o b s}$ and rate of events $R_{\text {evt }}$ for each detector. Right: net common observation time $T_{N}$ when at least $N_{\text {detectors }}$ were simultaneously operating.

\begin{tabular}{lcc||rc}
\hline Detector & $T_{\text {obs }}($ day $)$ & $R_{\text {evt }}($ events/day $)$ & $N_{\text {detectors }}$ & $T_{N}($ day $)$ \\
\hline ALLEGRO & 405.7 & 112.9 & 1 & 625.0 \\
AURIGA & 153.0 & 175.3 & 2 & 260.4 \\
EXPLORER & 137.5 & 150.7 & 3 & 89.7 \\
NAUTILUS & 108.5 & 80.8 & 4 & 15.5 \\
NIOBE & 185.9 & 14.0 & 5 & 0 \\
\hline
\end{tabular}

The amplitude distributions of all the 1997-1998 exchanged events for each detector are shown in Fig.2. The effective observation time of the exchanged data up to now is summarised in Table 2, together with the mean rate of exchanged events. The net observation time with at least four, three and two detectors simultaneously operating has been respectively $15.5,90$ and 260 days. We expect that the three-way observation time will increase significantly as the exchanged data set will become complete. The ALLEGRO detector has been showing the best duty cycle, close to $100 \%$ on the exchanged data period, as well as the most stationary noise performance with respect to the other detectors. 


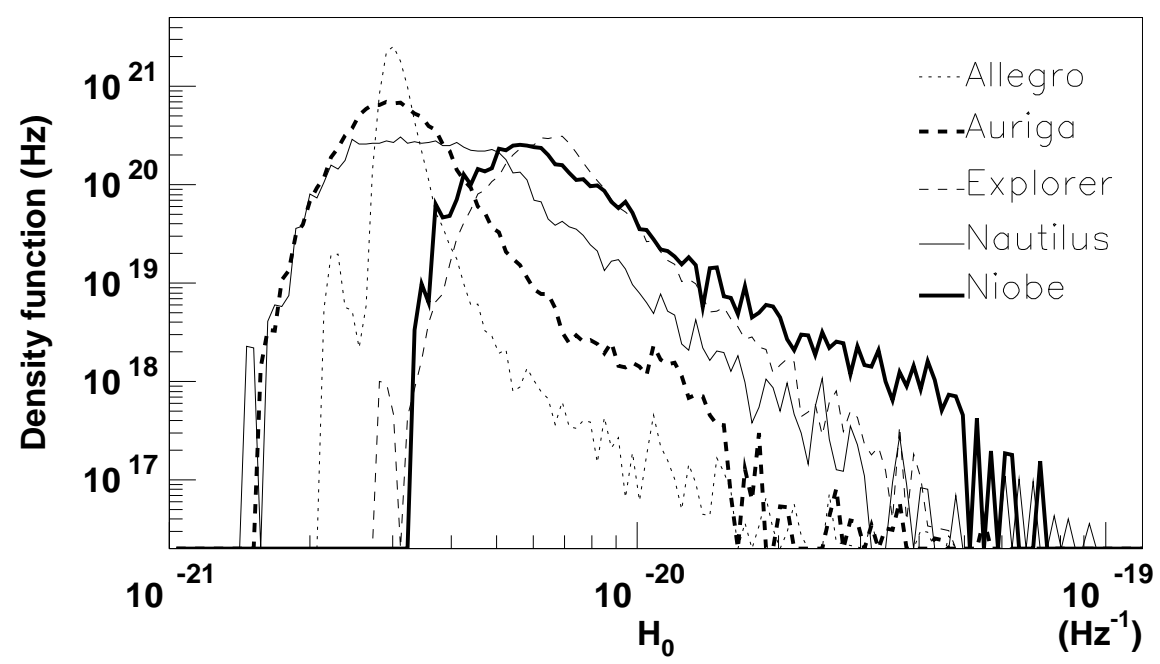

Fig. 2. Density functions of the amplitude of all the exchanged events in 1997-1998 for each detector. The densities are normalised to unit area and are estimated from the amplitude histograms of the events.

\section{Analysis of Time Coincidences}

A search for two, three and four-fold coincidences was carried out on the exchanged data. In the analysis reported here a $M$-fold coincidence is observed if the estimated arrival times $t_{i}$ at the $M$ detectors are all $\left|t_{i}-t_{j}\right| \leq 1 s$. This figure has been chosen as a compromise between the demands for a small accidental background and for a low false dismissal probability. ${ }^{\ddagger}$ In fact, the measured uncertainties $t_{w}$ on the estimated arrival times of a burst at each detector are quite similar and are selected to be $\pm 0.5 \mathrm{~s}$. This corresponds to a maximum false dismissal of a few $\%$ for the exchanged events, even at low $S N R$. The maximum separation among coincident events is therefore set to $2 t_{w}=1 s$.

A preliminary search for three and four-fold coincidences shows none. A detailed analysis is in progress. The two-fold coincidences found for each pair of detectors are shown in Table 3: in all the cases they correspond to the estimated accidental background.

Two methods for estimating the rate of accidental coincidences have been applied for the pairs of detectors: i) performing several time shifts of events times of one detector with respect to the other and then looking for coincidences? (the resulting accidental coincidences are reported as $\left\langle n_{a}\right\rangle$ in Tab.3); ii) assuming stationary Poisson distributions of event times and using the mean measured rates of events for each detector during the common observation time. The latter method predicts

\footnotetext{
$¥$ a new approach for multiple time coincidence analysis has been proposed by the Rome group.14
} 
Table 3. Preliminary results of two way coincidence analysis for the 1997-1998 data. The abbreviations, AL, AU, EX, NA and NI stand for ALLEGRO, AURIGA, EXPLORER NAUTILUS and NIOBE respectively. $n_{c}$ is the found number of coincidences, $\left\langle n_{a}\right\rangle$ and $\left\langle n_{a}\right\rangle_{\text {theory }}$ the expected accidental ones respectively from the time shift method and Eq. $P$, $P\left(n \geq n_{c} \mid<n_{a}>\right)$ the calculated probability for the coincidences to be $\geq n_{c}$, and $T_{o b s}$ the common observation time of the pairs of detectors.

\begin{tabular}{cccccc}
\hline Detectors & $n_{c}$ & $\left\langle n_{a}\right\rangle$ & $\left\langle n_{a}\right\rangle_{\text {theory }}$ & $P\left(n \geq n_{c} \mid\left\langle n_{a}\right\rangle\right)$ & $T_{o b s}$ \\
\hline AL-AU & 42 & 46.6 & 45.1 & 0.77 & 103.8 \\
AL-EX & 27 & 31.2 & 30.9 & 0.80 & 100.7 \\
AL-NA & 17 & 21.6 & 21.0 & 0.87 & 98.9 \\
AL-NI & 1 & 0.9 & 1.0 & 0.61 & 27.1 \\
AU-EX & 14 & 20.3 & 19.2 & 0.94 & 44.1 \\
AU-NA & 4 & 4.2 & 4.2 & 0.60 & 18.3 \\
AU-NI & 1 & 2.3 & 2.1 & 0.90 & 37.0 \\
EX-NA & 5 & 7.0 & 5.7 & 0.83 & 37.5 \\
EX-NI & 1 & 1.1 & 1.0 & 0.65 & 18.9 \\
\hline
\end{tabular}

a number of accidental $M$-fold coincidences given by 15

$$
<n_{a}>_{\text {theory }}=M \frac{\left(2 t_{w}\right)^{M-1}}{T_{o b s}^{M-1}} \prod_{i=1}^{M} n_{i},
$$

where $M$ is the number of detectors, $T_{o b s}$ their common observation time, $t_{w}$ the time window describing the timing accuracy of each detector, $n_{i}$ the number of exchanged candidate events of the $i^{\text {th }}$ detector during $T_{o b s}$. The agreement of both estimates of the accidental background of coincidences is well within the statistical uncertainties for the detector pairs. In addition, the observed coincidences, $n_{c}$, correspond to both estimates of the accidental coincidence background. This implies that no excess coincidences were observed.

Using Eq2, we also performed a preliminary analysis of the rates of accidental coincidences for three and four-fold configurations of the IGEC observatory. The most relevant result here is that the statistical significance of three-fold and four-fold time concidences among the current IGEC detectors improves by order of magnitudes.

\section{Future Plans and Conclusions}

This first IGEC joint analysis has shown that, with the current detector performances and the selected coincidence time window, at least three detectors simultaneously operating can perform an autonomous search for gw bursts with a very low false alarm rate even at signal-to-noise ratio as low as $3-5$ in single detectors. Therefore, the continuation of the IGEC international effort is very strongly motivated. Moreover, the joint work of the participating groups has instigated efforts to co-ordinate data analysis techniques between the different groups.

No coincidence above the expected accidental background were found in this preliminary analysis. Work is in progress to complete the analysis to three and four- 
fold coincidences, as well as to set upper limits on the rate of incoming gw bursts and on the amplitude of single gw bursts associated with selected time windows of astrophysical interest.

Improvements in the detector performances will lead to increase the sensitivity of the IGEC observatory in two respects. On one side the thresholds for gw burst search will be lowered without increasing the level of the accidental background rate. On the other side the effective bandwidths of the detectors will be also widened, thus decreasing the uncertainty in the estimated arrival time of a gw burst. The latter will allow the lowering of the rate of accidental coincidences and, above all, it will give the opportunity to measure the propagation speed and direction of the incoming gw.

In this framework, we think that the participation within the IGEC observatory of the interferometric detectors as they will begin observations would constitute a very important stage towards the establishment of the future worldwide observatory for gravitational waves.

\section{Acknowledgements}

The ALLEGRO group was supported by the National Science Foundation and LSO, the NIOBE group by the Australian Research Council. The Italian groups were supported in part by a grant from MURST-COFIN'97. The Rome group thanks F.Campolungo, G.Federici, R.Lenci, G.Martinelli, E.Serrani, R.Simonetti and F.Tabacchioni for their precious technical assistance.

\section{References}

1. T. Nakamura et al., Astrophys. J. 487 (1997) L139.

2. E. Amaldi et al., Astron. Astroph. 216 (1989) 325.

3. P. Astone et al., Phys. Rev. D 59 (1999) 122001.

4. E. Mauceli et al., Phys. Rev. D 54 (1997) 1264.

5. G.A. Prodi et al., in Proc. of the Second Edoardo Amaldi Conference, ed. E. Coccia et al. (World Scientific, Singapore, 1998) pp. 148-158.

6. P. Astone et al., Phys. Rev. D 47 (1993) 362; from 1998 Explorer is a CERN Recognized Experiment.

7. P. Astone et al., Astroparticle Phys. 7 (1997) 231.

8. D.G. Blair et al., Phys. Rev. Lett. 74 (1995) 1908.

9. see for instance: http://igec.lnl.infn.it

10. S. Vitale et al., Nucl. Phys. B48 (1996) 104-106.

11. P. Astone et al., Nuovo Cimento C 20 (1997) 9-60

12. L. Baggio et al., Phys. Rev. D (2000) in press.

13. G.A. Prodi et al., in Proc. of the Third Edoardo Amaldi Conference (Pasadena, 1999), ed. S. Meshkov et al., in press.

14. R. Terenzi, N-fold time coincidences on IGEC Gravitational Wave events data using Data Base technology, these Proceedings.

15. see for instance R.K.Bock et al., in Data Analysis techniques for high-energy physics experiments, Cambridge University Press (1990) p. 22. 